Keywords: Antidepressants; Register-based study;

\title{
Use of antidepressants among 0-26 year olds in Finland during 1997-2007
}

\author{
Ilona Autti-Rämö* \\ Andre Sourander** \\ Johanna Seppänen*** \\ Jaana E. Martikainen ${ }^{\star \star \star \star}$ \\ * The Social Insurance Institution, \\ Research Department, \\ Finland \\ ** Turku University and Turku \\ University Hospital, \\ Finland \\ *** Finnish Institute of Occupational Health, \\ Statistics and Health Economics, \\ Finland

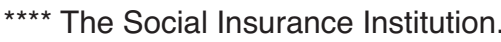 \\ Research Department, \\ Finland \\ FINLAND
}

\begin{abstract}
Background and Objectives: The use of antidepressants has increased in many Western countries among children, adolescents and young adults. The objective of this study was to analyse changes in incidence and prevalence of antidepressant use among patients aged 0 to 26 years from year 1997 to 2007.

Methods: This is an observational population-based drug utilization study using a nationwide prescription register including data on reimbursed purchases of antidepressants for outpatient use.

Results: The incidence and prevalence of antidepressant use increased about three fold during the study period. Among children less than 14 years of age both incidence and prevalence were low during the study period. In the age group 14-26 years, the incidence increased from 6.4 to 16.1 (per 1000 person-years) among men and from 9.7 to 28.3 among women, while the prevalence went up from 10.4 to 30.4 among men and from 15.5 to 56.8 among women. By the age of 26 years, $11.6 \%$ of men and $17.8 \%$ of women had purchased antidepressants at least once. The first antidepressant prescriptions were mainly prescribed by a specialist for children aged 7 to 15 years and by a non-specialist for the older age groups.
\end{abstract}


Conclusions: Use of antidepressants has increased remarkably among adolescents and young adults during recent years. Further studies are warranted to analyse whether the increase in use is due to an increase in depression incidence, a lower threshold of prescribing medications or a lack of psychosocial treatment resources.

Received: 16 January 2010

Revised: 22 March 2011

Accepted: 1 April 2011

\section{Introduction}

The use of antidepressants has increased in many Western countries among children, adolescents and young adults, and it has been the subject of numerous studies and a topic of concern ${ }^{1-6}$. This increase is mainly due to the introduction of new antidepressants which were anticipated to have fewer side effects, lower toxicity in overdose, and a broader range of treatment indications than older antidepressants. Concerns about the safety of antidepressant use among children and youth were raised in 2003 as the manufacturer of Paxil (paroxetine) notified the U.S. Food and Drug Administration (FDA) of a clinical trial indicating an increased risk of suicidal thoughts and actions in children and adolescents ${ }^{7}$. In 2004 the FDA required a black-box warning on antidepressants that describes a possible increase in suicidality risk in children and adolescents $^{8}$. In 2007 the warning was extended to young adults of up to 24 years of age. The FDA emphasized the need for appropriate monitoring and close observation after initiation of antidepressant medication.

There is robust evidence that after the warning the use of antidepressants in children declined significantly in the United States ${ }^{7}$. A shift was also detected in the diagnosing of depression in children by a psy- chiatrist rather than a primary care provider ${ }^{9}$. There are, however, large variations in the prevalence of antidepressant use between various countries ${ }^{3}$ and ethnic groups in the United States ${ }^{6}$.

The present study examines the yearly prevalence and incidence of antidepressant use in Finland among individuals 0-26 years old from 1997 to 2007 using data obtained from the nationwide individual based Prescription Registry maintained by the Social Insurance Institution (SII). In addition, we estimated the cumulative incidence of antidepressant use in one birth cohort, those aged 26 years in 2007. Finally we analysed the speciality of the doctor initiating the antidepressant treatment in 2007 in order to understand the current practice in Finland.

\section{Methods}

The SII maintains a national Prescription Register containing data on reimbursed medication purchases. The reimbursement system covers all permanent residents living in Finland, while the Register encompasses about $90 \%$ of the antidepressants sold through Finnish pharmacies. The Register includes the patient's personal identification number, the Anatomical Therapeutic Chem- 
ical (ATC) $\operatorname{code}^{10}$ of the dispensed medicine, the date of dispensing, and the unique identifier of the prescribing doctor.

The study material consisted of reimbursed antidepressant drug purchases (ATC classes N06A and N06C) in age group 0-26 during the years 1996-2007. The observation period was 1997-2007, but data from the year 1996 was collected to provide the incidence calculations for the year 1997. For purposes of subgroup analysis, the antidepressants were classified into three groups: 1) selective serotonin reuptake inhibitors (SSRIs, ATC class N06AB) 2) tricyclic antidepressants (N06AA and N06CA), and 3) other antidepressants (N06AF-AX).

An incident antidepressant user during a calendar year was defined as a person i) who had made at least one purchase of antidepressants during the calendar year, and ii) whose most recent purchase had been made more than 365 days ago. Similarly, a prevalent user was defined as a person who had made at least one antidepressant drug purchase during the calendar year. The yearly incidence and prevalence of antidepressant use were calculated as the number of incident or prevalent users divided by the number of inhabitants in the corresponding age group (residence status as of $31^{\text {th }}$ December each year).

An incident user who made no further antidepressant purchases during the following 365 days was defined as a single user.

Our data did not enable us to estimate the lifetime prevalence of antidepressant use. To demonstrate the cumulative incidence of antidepressant drug use, the incidence in a cohort aged 26 years in 2007 (born in 1981) was cumulated over ages 16-26 (from year 1996 to 2007). To calculate the cumulative incidence, each user was counted only once.
The initiator of antidepressant medication for new patients in 2007 was captured from the Health Care Personnel Register, which, too, is maintained by the SII. For the purpose of analysis, the doctors were classified in six groups according to their speciality: 1) specialists in child or adolescent psychiatry, 2) other psychiatrists (general, forensic), 3) pediatricians or pediatric neurologists, 4) specialists in general practice, 5) other specialities, and 6) no specialty. If a doctor had several specialities, he or she was classified according to the field that was ranked highest in the list above. In this analysis, a new user was defined as a person who had made no antidepressant purchases during years 1996-2007.

The results on yearly incidence and prevalence of antidepressant use are reported for the years 1997, 2002 and 2007 by age and sex. In Appendix 1 the results are reported by sex and by age group (0-6 years, 7-10 years, $11-15$ years, $16-20$ years and 21 26 years) for the year 2007. These age groups were also used when analysing the speciality of the doctor initiating the antidepressant medication in year 2007 .

This study has been approved by the Ethical Committee of the SII.

\section{Results}

In the Prescription Register, there were a total of 971,266 antidepressant drug purchases made by 122,792 patients aged 0-26 years during the years 1996-2007.

Both the yearly incidence and prevalence of antidepressant use increased during the study period (Figure 1 and Appendix 1). In the whole study population, the incidence of use increased during years 1997-2007 from 
a) Prevalence (1/1000), men



c) Incidence (1/1000), men

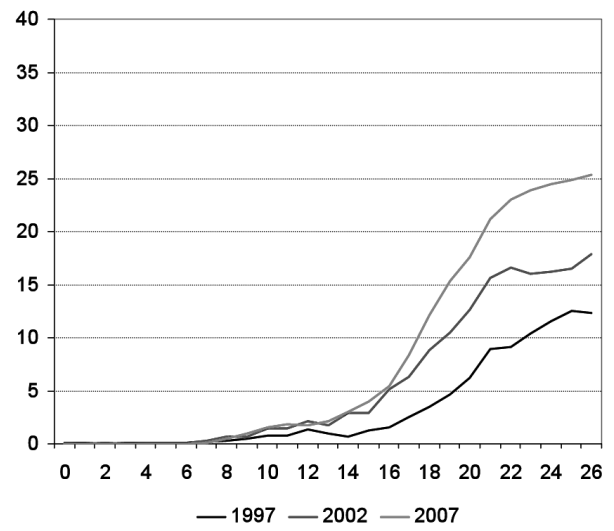

b) Prevalence (1/1000), women

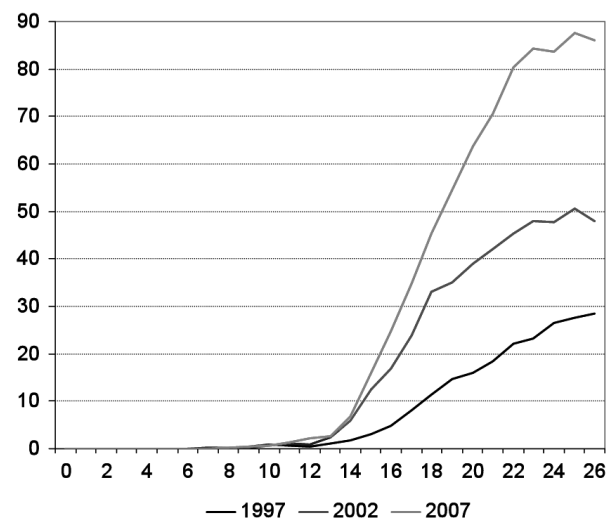

d) Incidence (1/1000), women

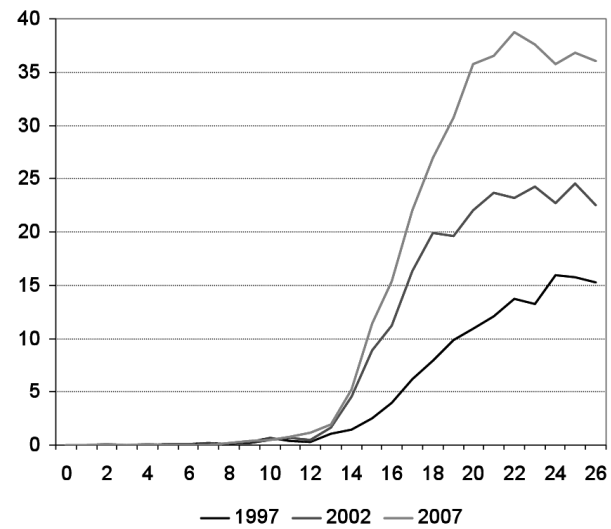

Figure 1. The incidence and prevalence of antidepressant use increased during the study period.

3.3 to 8.5 (per 1000 person-years) among men and from 4.8 to 14.6 among women. Corresponding change in prevalence was from 5.3 to 16.0 among men and from 7.6 to 29.2 among women. Among children younger than 14 years, both incidence and prevalence levels remained low during the whole study period. In the age group 14-26 years, the incidence increased from 6.4 to 16.1 among men and from 9.7 to 28.3 (per 1000 person-years) among women, while the prevalence rose from 10.4 to 30.4 among men and from 15.5 to 56.8 among women.
The proportion of single time users declined steadily during the study period. In $1997,30.7 \%$ of men and $26.6 \%$ of women among the incident users purchased antidepressants only once. In 2007, the corresponding proportions were $26.0 \%$ and $20.9 \%$.

For the birth cohort of 1981 the cumulated incidence of antidepressant use at ages 16 to 26 years among women was 178.1 , indicating that $17.8 \%$ of the women aged 16 years in 1997 purchased antidepressants at least once prior to their $27^{\text {th }}$ birthday. The 
corresponding figure for men was $11.6 \%$ (see also Appendix 2).

A trend analysis by subgroups of antidepressants showed that the increase in antidepressant use was due to elevated use of newer antidepressants. During the study period, prevalence of the use of tricyclic antidepressants decreased slightly from 1.2 to 1.1 (per 1000 person-years) while prevalence of the use of SSRIs increased from 5.1 to 17.9 and of other antidepressants from 0.8 to 6.2 .

The speciality of the doctor who prescribed the very first antidepressant varied by patient's age (Table 1). Antidepressant prescriptions for children under the age of 7 were rare. Doctors who had specialized in some field of psychiatry initiated the majority of the medications for patients aged 7-15 years. For older patients, medication was most often initiated by a doctor without specialization. $39 \%$ of the patients aged 16-20 years and $21 \%$ of those aged 21-26 years received their first antidepressant prescription from a doctor who had a speciality within psychiatry.

\section{Discussion}

Both the incidence and prevalence of antidepressant use increased during the study period, especially for women and for newer antidepressants. Nearly one in five women and one in ten men had purchased antidepressants prior to the age of 27 years. The first antidepressant prescription of those aged 16 years or more was most often ordered by an unspecialized doctor, in contrast to the younger age groups whose treatment was mainly initiated by specialists.

The national prescription register enabled us to retrospectively determine nearly all purchases of antidepressants from year 1996 on and to analyse the data at a patient level and at a prescribing doctor level. The coverage of the prescription register was good during the whole study period. The prescription register does not contain information about the indication for a prescription and thus we were not able to analyse the treatment indications.

Time trend analyses of nationwide individual based register data are few. Methodological heterogeneity and differences in age grouping and time frames in studies assessing the use of antidepressants make comparisons difficult. Yet it seems that the use of antidepressants among children, adolescents and young adults remains quite low in Finland. In a multinational comparison from 2000, the one-year prevalence of antidepressant use among children under age 15 was multifold in the USA compared with the Netherlands, Denmark and Germany ${ }^{3}$. The differences were somewhat smaller yet evident in older age groups. In our study very few children under the age of 14 used antidepressants even in 2007. A comparison between Scandinavian countries in 2003 showed that the use of antidepressants among children and adolescents in Finland was at about the same level as in Denmark and lower than in Iceland and Sweden ${ }^{11}$. The FDA warning concerning the use of SSRIs for children and adolescents did not affect antidepressant use in Finland ${ }^{12}$. To understand the reason for this further research is required.

Prior epidemiological studies show up to $25 \%$ lifetime prevalence of major depressive disorder by the end of adolescence ${ }^{13-16}$. By using one birth cohort we were able to estimate that nearly one in five women and one in ten men had bought antidepressants prior to the age of 27 . The majority of first prescriptions in the age range 16-26 years 


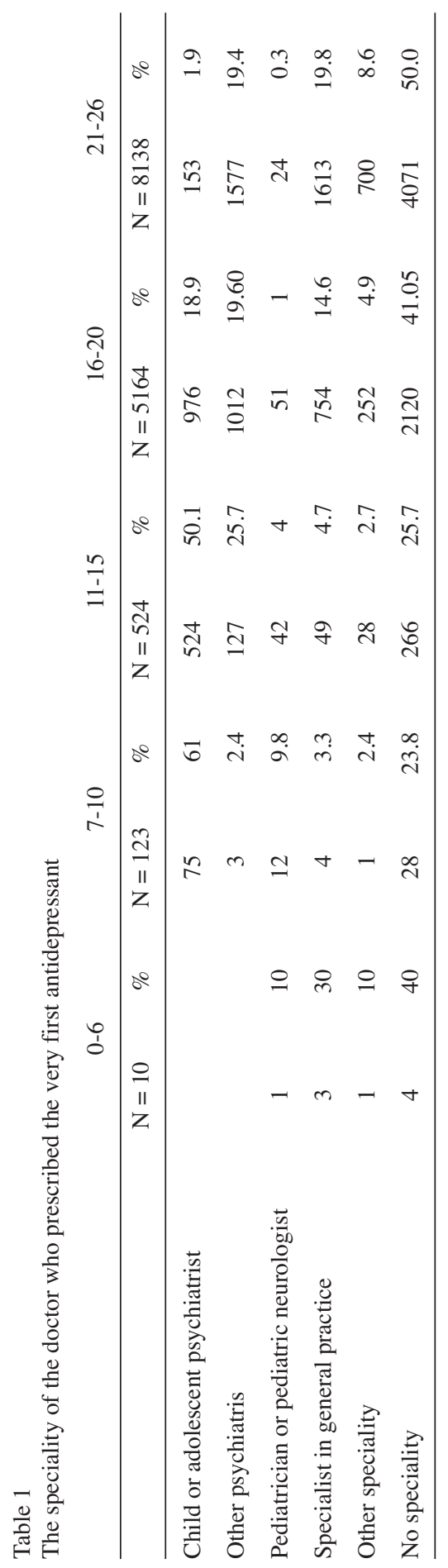

were written by non-specialists. It can be presumed that the increase in the use of antidepressants in the Finnish population may at least partly be based on wider treatment indications as observed in the Netherlands ${ }^{4}$ and/or a lower threshold for the use of such drugs, and not solely on previously unmet needs. Psychotropic prescribing practices may differ between countries for several reasons, including differences in cultural background, professional education, resources at psychosocial treatment facilities, drug regulation and health care systems $s^{2,6,17,18}$.

Both the newly updated Finnish evidence-based guideline on treating depression in adolescents and young adults ${ }^{19}$ and the FDA medication guide emphasize close observation after initiation of SSRI medication $^{8}$. It is of concern whether the resources of the Finnish health care system -as regards its ability to provide follow-up for medication and psychosocial support with therapeutic interventions when needed- will stretch to meet the demands of good, evidence-based treatment of depression, anxiety and other disorders treated with antidepressants given the observed increased incidence of antidepressant use. This concern is shared universally. Prescribing an antidepressant is not the end but the beginning of the treatment relationship.

\section{Acknowledgements}

We thank Hilkka Ruuska for her assistance with data analysis and research professor Raimo Raitasalo for his help in designing the study. 


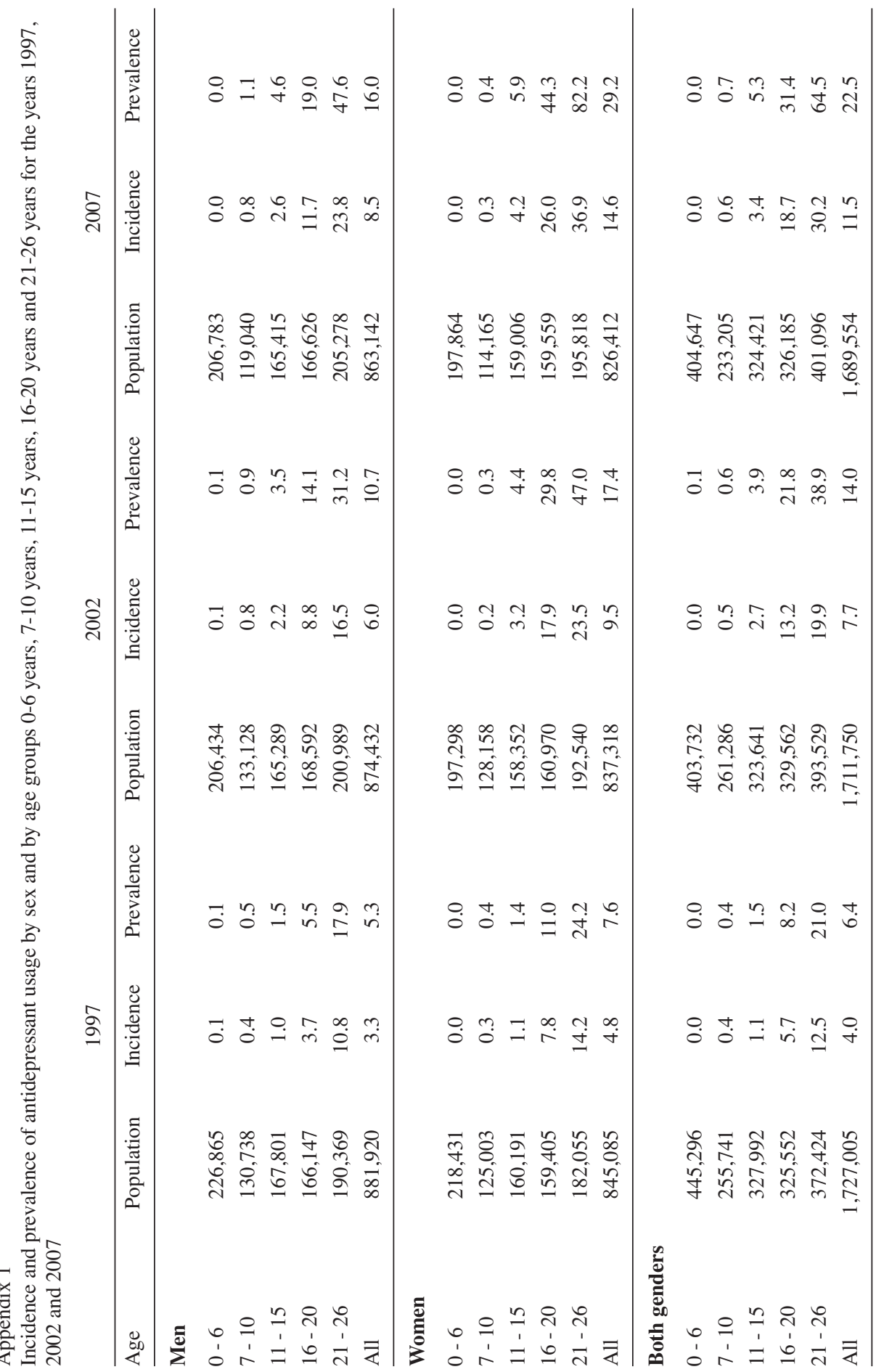


Appendix 2

Cumulative incidence (per 1000) of antidepressant use for the ages 16-26 years for the cohort born in 1981

\begin{tabular}{crrr} 
Age & Men & Women & Both genders \\
\hline 16 & 2.5 & 4.0 & 3.2 \\
17 & 4.5 & 8.9 & 6.6 \\
18 & 8.3 & 18.5 & 13.2 \\
19 & 14.6 & 34.4 & 24.2 \\
20 & 23.0 & 51.7 & 37.0 \\
21 & 34.2 & 69.5 & 51.4 \\
22 & 46.1 & 87.2 & 66.0 \\
23 & 59.5 & 108.0 & 83.1 \\
24 & 74.0 & 130.4 & 101.4 \\
25 & 89.4 & 153.7 & 120.6 \\
26 & 106.5 & 176.9 & 140.7 \\
\hline
\end{tabular}

\section{References}

1. Hunkeler EM, Fireman B, Lee J, Diamond R, Hamilton J, He CX, et al. Trends in use of antidepressants, lithium, and anticonvulsants in Kaiser Permanente-insured youths, 1994-2003. J Child Adolesc Psychopharmacol 2005; 15(1): 26-37.

2. Fegert JM, Kolch M, Zito JM, Glaeske G, Janhsen K. Antidepressant use in children and adolescents in Germany. J Child Adolesc Psychopharmacol 2006; 16(1-2): 197-206.

3. Zito JM, Tobi H, de Jong-van den Berg LT, Fegert JM, Safer DJ, Janhsen K, et al. Antidepressant prevalence for youths: a multi-national comparison. Pharmacoepidemiol Drug Saf 2006; 15(11): 793-798.

4. Volkers AC, Heerdink ER, van Dijk L. Antidepressant use and off-label prescribing in children and adolescents in Dutch general practice (2001-2005). Pharmacoepidemiol Drug Saf 2007; 16(9): 1054-1062.

5. Olfson M, Marcus SC, Weissman MM, Jensen PS. National trends in the use of psychotropic medications by children. J Am Acad Child Adolesc Psychiatry 2002; 41(5): 514-521.

6. Olfson M, Marcus SC. National patterns in antidepressant medication treatment. Arch Gen Psychiatry 2009; 66(8): 848-856.

7. Busch SH, Barry CL. Pediatric antidepressant use after the black-box warning. Health Aff (Millwood) 2009; 28(3): 724-733.
8. U.S. Food and Drug Administration. Antidepressant Use in Children, Adolescents, and Adults. Available at: http://www.fda.gov/Drugs/DrugSafety/InformationbyDrug Class/ucm096273.htm.

9. Bhatia SK, Rezac AJ, Vitiello B, Sitorius MA, Buehler BA, Kratochvil CJ. Antidepressant prescribing practices for the treatment of children and adolescents. J Child Adolesc Psychopharmacol 2008; 18(1): 70-80.

10. WHO Collaborating Centre for Drug Statistics Methodology. Available at: http://www.whocc.no/.

11. Medicines consumption in the nordic countries 1999-2003. Copenhagen: NOMESCO (Nordic medical statistical committee); 2004. p. 101.

12. Foulon V, Svala A, Koskinen H, Chen TF, Saastamoinen LK, Bell JS. Impact of regulatory safety warnings on the use of antidepressants among children and adolescents in Finland. J Child Adolesc Psychopharmacol 2010; 20(2): 145-150.

13. Lewinsohn PM, Hops H, Roberts RE, Seeley JR, Andrews JA. Adolescent psychopathology: I. Prevalence and incidence of depression and other DSM-III-R disorders in high school students. J Abnorm Psychol 1993; 102(1): 133-144.

14. Kessler RC, Avenevoli S, Ries Merikangas K. Mood disorders in children and adolescents: An epidemiologic perspective. Biol Psychiatry 2001; 49(12): 1002-1014.

15. Costello EJ, Mustillo S, Erkanli A, Keeler G, Angold A. Prevalence and development of psychiatric disorders in childhood and adolescence. Arch Gen Psychiatry 2003; 60(8): 837-844. 
16. Fergusson DM, Horwood LJ, Ridder EM, Beautrais AL. Suicidal behaviour in adolescence and subsequent mental health outcomes in young adulthood. Psychol Med 2005; 35(7): 983-993.

17. Vitiello B, Zuvekas SH, Norquist GS. National estimates of antidepressant medication use among U.S. children, 1997-2002. J Am Acad Child Adolesc Psychiatry 2006; 45(3): 271-279.

18. Vitiello B. An international perspective on pediatric psychopharmacology. Int Rev Psychiatry 2008; 20(2): 121-126.

19. Suomalaisen Lääkäriseuran Duodecimin ja Suomen Psykiatriyhdistys ry:n asettama työryhmä. Depression (in
Finnish). 2009; Available at: www.kaypahoito.fi/kh/kaypahoito?suositus=hoi50023. Accessed 2nd september, 2009.

Author for correspondence:

Ilona Autti-Rämö

The Social Insurance Institution

Research Department

PO Box 450, FI-00101

Helsinki

FINLAND

Email: ilona.autti-ramo@kela.fi 